\title{
Common Fixed Point Theorem in Intuitionistic Fuzzy Metric Spaces Satisfying Integral Type Inequality Varun Singh ${ }^{1}$, Arvind Gupta ${ }^{2}$, Geeta Modi ${ }^{3}$ \\ ${ }^{1}$ Research Scholar Govt. M.V.M. Bhopal, MP, India \\ ${ }^{2}$ Professor Department of Mathematics, Govt. M.V.M. Bhopal, MP, India. \\ ${ }^{3}$ Head \& Professor Department of Mathematics, Govt. M.V.M. Bhopal, MP, India.
}

Keywords- Intuitionistic fuzzy metric space, non compatible maps, weakly compatible maps, common fixed point, EA property.

Abstract: We prove common fixed point theorem for weakly compatible maps in Intuitionistic fuzzy metric space satisfying integral type inequality but without using the completeness of space or continuity of the mappings involved. We prove by using the concept of E A property.

\section{Introduction}

In the study of common fixed points of compatible mappings we often require assumption on completeness of the space or continuity of mappings involved besides some contractive condition but the study of fixed points of non comapatible mappings can be extend to the class of non expansive or Lipschitz type mapping pairs even without assuming the continuity of the mappings involved or completeness of the space. Aamri and El Moutawakil [6] generalized the concepts of non comapatibility by defining the notion of (E.A) property and proved common fixed point theorems under strict contractive condition.

We prove common fixed point theorems for weakly compatible maps in Intuitionistic fuzzy metric space by using the concept of (E.A) property, however, without assuming either the completeness of the space or continuity of the mappings involved.

\section{Preliminaries}

Definition:- A binary operation $*:[0,1] \times[0,1] \rightarrow[0,1]$ is continuous t-norm if is satisfying the following condition:

(i) * is commutative and associative ;

(ii) $*$ is continuous ;

(iii); $\quad a * 1=a$ for some $a \in[0,1]$;

(iv) $\quad a * b \leq c * d$ whenver $a \leq c$ and $b \leq d$ and $a, b, c, d \in[0.1]$

Definition:- A binary operation ${ }^{0}:[0,1] x[0,1] \rightarrow[0,1]$ is continuous t-conorm if ${ }^{\circ}$ is satisfying the following condition:

(i) ${ }^{\circ}$ is commutative and associative ;

(ii) ${ }^{\mathrm{o}}$ is continuous ;

(iii) $\mathrm{a}^{\mathrm{o}} 0=\mathrm{a}$ for some $\mathrm{a} \in[0,1]$

(iv) $\mathrm{a}^{\mathrm{o}} \mathrm{b} \leq \mathrm{c}^{\mathrm{o}} \mathrm{d}$ whenever $\mathrm{a} \leq \mathrm{c}$ and $\mathrm{b} \leq \mathrm{d}$ and $\mathrm{a}, \mathrm{b}, \mathrm{c}, \mathrm{d} \in[0,1]$

Definition:-The 5- tuple $\left(\mathrm{X}, \mathrm{M}, \mathrm{N},{ }^{*},{ }^{\circ}\right)$ is said to be an Intuitionistic Fuzzy Metric Space if $\mathrm{X}$ is an arbitrary non empty set, ${ }^{*}$ is a continuous t-norm, ${ }^{\circ}$ is a continuous t-conorm and $\mathrm{M}, \mathrm{N}$ are a Fuzzy set on $\mathrm{X}^{2} \times[0,1]$ satisfying the following conditions:

For all $\mathrm{x}, \mathrm{y}, \mathrm{z}, \in \mathrm{X}, \mathrm{s}, \mathrm{t}>0$ 
(i) $M(x, y, t)+N(x, y, t) \leq 1$;

(ii) $M(x, y, 0)>0$;

(iii) $M(x, y, t)=1$ for all $t>0$ iff $x=y$;

(iv) $M(x, y, t)=M(y, x, t)$;

(v) $M(x, y, t) * M(y, z, s) \leq M(x, z, t+s)$;

(vi) $M(x, y, \cdot):(0, \infty) \rightarrow(0,1]$ is continuous;

(vii) $N(x, y, 0)>0$;

(viii) $N(x, y, t)=0$ for all $t>0$ iff $x=y$;

(ix) $N(x, y, t)=N(y, x, t)$;

(x) $N(x, y, t) \circ N(y, z, s) \leq N(x, z, t+s)$;

(xi) $N(x, y):,(0, \infty) \rightarrow(0,1]$ is continuous;

Example: - Let $(X, d)$ be a metric space. Define $a * b=a b$ and

$a \circ b=\min \{1, a+b\}$ for all $a, b \in[0,1]$ and let $\mathrm{M}$ and $\mathrm{N}$ be fuzzy sets on $\mathrm{X}^{2} \times[0,1]$ defined as follows :

$M(x, y, t)=\frac{t}{t+d(x, y)}$

$$
N(x, y, t)=\frac{d(x, y)}{t+d(x, y)}
$$

Then $(X, M, N, *, \bullet)$ is an intuitionistic fuzzy metric space.

Definition: - Let $U$ and $V$ be two self maps of an intuitionistic fuzzy metric space

$(X, M, N, *, \diamond) U$ and $V$ are said to be compatible if $M\left(U V x_{n}, V U x_{n}, t\right) \rightarrow 1$ and $N\left(U V x_{n}, V U x_{n}, t\right) \rightarrow 0$ as $n \rightarrow \infty$ whenever $\left\{\mathrm{x}_{n}\right\}$ is a sequence in $\mathrm{X}$ such that $U V x_{n}, V U x_{n} \rightarrow z$ as $n \rightarrow \infty \quad$,for some $z \in X$

Definition :- Two self maps $U$ and $\mathrm{V}$ of intuitionistic fuzzy metric space $(X, M, N, *, 0)$ are said to be weakly compatible if they commute at their coincidence point, i.e. $U V x=V U x$ whenever $U x=V x \quad x \in X$.

Clearly each pair of compatible self maps is weakly compatible but the converse is not true always.

Definition:-Let $\mathrm{A}$ and $\mathrm{B}$ be two self-maps of a intuitionistic fuzzy metric space $(X, M, N, *, 0)$. We say that $A$ and $B$ satisfy the property (E.A) if there exists a sequence $\left\{x_{n}\right\}$ such that $\lim _{n \rightarrow \infty} A x_{n}=\lim _{n \rightarrow \infty} B x_{n}=z$ for some $z \in X$

Note that weakly compatible and property (E.A) are independent to each other. 
Definition: - Let $\mathrm{f}$ and $\mathrm{g}$ be two self maps of a metric space $(X, d)$ and $f$ and $g$ to be weakly commuting if

$d(f g x, g f x) \leq d(g x, f x)$ for all. $x \in X$

It can be seen that commuting maps ( $f g x=g f x \forall x \in X)$ are weakly compatible, but converse is false.

In 2002, A.Branciari[1] analyzed the existence of fixed point for mapping $\mathrm{T}$ defined on a complete metric space $(X ; d)$ satisfying a general contractive condition of integral type in the following theorem.

Theorem[1] :- Let $(X ; d)$ be a complete metric space, $c \in(0,1)$ and let $T: X \rightarrow X$ be a mapping such that for each $x, y \in X$,

$$
\int_{0}^{\mathrm{d}(\mathrm{Tx}, \mathrm{Ty})} \varphi \mathrm{dt} \leq c \int_{0}^{\mathrm{d}(\mathrm{Tx}, \mathrm{Ty})} \varphi \mathrm{dt}
$$

where $\varphi[0,+\infty) \rightarrow[0 ;+\infty i$ s a Lesbesgue-integrable mapping which is summable (i.e. with finite integral) on each compact subset of $[0 ;+\infty)$, non-negative, and such that for each

$$
\varepsilon>0, \quad \int_{0}^{\varepsilon} \varphi \mathrm{dt} \geq 0
$$

then $\mathrm{T}$ has a unique fixed point $a \in X$ such that for each $x \in X$,

$$
\lim _{\mathrm{n} \rightarrow \infty} \mathrm{T}^{\mathrm{n}} \mathrm{x}=\mathrm{a}
$$

After the paper of Branciari[1], a lot of research works have been carried out on generalizing contractive conditions of integral type for different contractive mappings satisfying various known properties. A fine work has been done by Rhoades[2] extending the result of Branciari[1] by replacing the condition (i) by the following

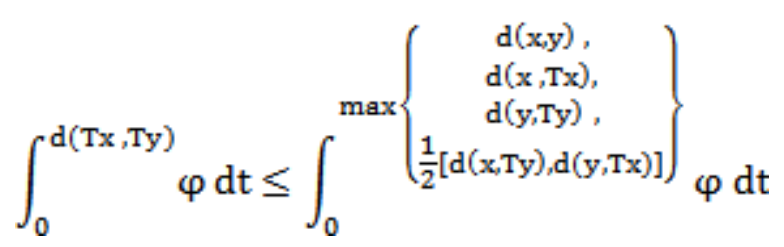

\section{Main Results run as}

Theorem:- Let $f$ and $g$ be two weak - compatible self maps of intuitionistic fuzzy metric $(X, M, N, *, 0)$ space satisfying the property E.A. and

$$
f X \subset g X
$$

$$
\begin{aligned}
\int_{0}^{M(f x, f y, k t)+N(f x, f y, k t)} \emptyset(\mathrm{t}) \mathrm{dt} \geq & \int_{0}^{M(g x, g y, t)+N(g x, g y, t)} \emptyset(\mathrm{t}) \mathrm{dt}, \mathbf{k} \geq \mathbf{0} \\
& \min \left\{\begin{array}{c}
M(f f x, g x, t)+N(f f x, g x, t), \\
M(g g x, f x, t)+N(g g x, f x, t), \\
M(f f x, g g x, t)+N(f f x, g g x, t)
\end{array}\right\} \emptyset(\mathrm{t}) \mathrm{dt}
\end{aligned}
$$

Whenever $f x \neq f^{2} x$

If the range of $f$ or $g$ is complete subspace of $X$, then $f$ and $g$ have a common fixed point 


\section{Proof:-}

Since $f$ and $g$ are satisfy the property E.A., so there exists a sequence $\left\{\mathrm{x}_{\mathrm{n}}\right\}$ in $\mathrm{X}$ such that $f \mathrm{x}_{\mathrm{n}}, \mathrm{gx}_{\mathrm{n}} \rightarrow \mathrm{z}$ as $\mathrm{n} \rightarrow \infty$ for $\mathrm{z} \in \mathrm{X}$

Since $z \in f X$ and $\subset g X$, there exists some point $s$ in $X$ such that

$$
z=g s \text { where } g x_{n} \rightarrow z \text { as } n \rightarrow \infty
$$

If then

$$
f s \neq g s
$$

$$
\int_{0}^{M\left(f x_{n}, f s, k t\right)+N\left(f x_{n}, f s, k t\right)} \emptyset(\mathrm{t}) \mathrm{dt} \geq \int_{0}^{M\left(g x_{n}, g s, t\right)+N\left(g x_{n}, g s, t\right)} \emptyset(\mathrm{t}) \mathrm{dt}
$$

Taking limit $n \rightarrow \infty$ we get

Not possible

$$
\begin{aligned}
\int_{0}^{M(g s, f s, k t)+N(g s, f s, k t)} \emptyset(\mathrm{t}) \mathrm{dt} & \geq \int_{0}^{M(g s, g s, t)+N(g s, g s, t)} \emptyset(\mathrm{t}) \mathrm{dt} \\
& =\int_{0}^{1} \emptyset(\mathrm{t}) \mathrm{dt}
\end{aligned}
$$

Hence $\quad f s=g s$

Since ${ }^{f}$ and $g$ are weakly compatible so $=g f s$, therefore $f g s=f f s=g f s=g g s$ If $f f s \neq f s$ then

$$
\begin{aligned}
& \int_{0}^{M(f s, f f s, t)+N(f s, f f s, t)} \emptyset(\mathrm{t}) \mathrm{dt}>\int_{0} \min \left\{\begin{array}{c}
M(f f s, g s, t)+N(f f s, g s, t), \\
M(g g s, f s, t)+N(g g s, f s, t), \\
M(f f s, g g s, t)+N(f f s, g g s, t)
\end{array}\right\} \emptyset(\mathrm{t}) \mathrm{dt} \\
& =\int_{0} \min \left\{\begin{array}{c}
M(f f s, g s, t)+N(f f s, g s, t), \\
M(g g s, f s, t)+N(g g s, f s, t), \\
M(f f s, f f s, t)+N(f f s, f f s, t)
\end{array}\right\} \emptyset(\mathrm{t}) \mathrm{dt} \\
& =\int_{0}^{\min \left\{\begin{array}{c}
M(f f s, f s, t)+N(f f s, f s, t), \\
M(f f s, f s, t)+N(f f s, f s, t), \\
1+0
\end{array}\right\}} \emptyset(\mathrm{t}) \mathrm{dt} \\
& =\int_{0}^{M(f f s, f s, t)+N(f f s, f s, t)} \emptyset(\mathrm{t}) \mathrm{dt}
\end{aligned}
$$

This is contradiction and so $f f s=f s$

$\Rightarrow f s=f f s=f g s=g f s=g g s$

Hence $f s$ is a common fixed point of $f$ and $g$.

The case when $f X$ is a complete subspace $X$ is similar to the above since . $f X \subset g X$

\section{Conclusion}

We prove fixed point theorem for weakly compatible maps in intuitionistic fuzzy metric space satisfying integral type inequality by using E.A. property. 


\section{References}

[1]. A. Branciari (2002), "A fixed point theorem for mappings satisfying a general contractive condition Of integral type" IJMMS 29:9, 531-536.

[2] B.E Rhoades (2003)., "Two fixed point theorem for mapping satisfying a general contractive condition of integral type". Int. J. Math. Sci., 3: 4007-4013.

[3] B.E. Rhoades and G. Jungck (1998), "Fixed points for set valued function without continuity", Indian J. Pure Appl. Math. 29(3), 227-238.

[4] G. Jungck (1986), “Compatible mappings and common fixed points", Int. J.Math. \& Math. Sci. 9, 771-779.

[5] Grabiec, M. (1988), Fixed Point in Fuzzy Metric Spaces , Fuzzy Sets and Syestems $27385-$ 389.

[6] M. Aamri and D. El Moutawakil (2002), "Some new common fixed point theorem under strict contractive conditions” J. Math. Anal. Appl.,270, 181-188.

[7] Mantu saha(2012), "Fixed point theorems for A-contraction mappings of integral type" J. Nonlinear science and application, 5, 84-92.

[8] Park . "Intuitionistic fuzzy metric space." Chaos, Solitons and Fractals 2004;22;1039-46

[9] S.N. Mishra (1994), N. Sharma and S.L. Singh, "Common fixed points of maps on fuzzy metric space", Int. J. Math. \& Math. Sci. 17, 253-258.

[10] Schweizer B,Sklar A. “Statistical metric spaces.” Pacific J. Math 1960; 10;314-334

[11] Zadeh, (1965), L. A., "Fuzzy sets, Inform. Control” 8 ,338-353 